\title{
The Internal Job Market of the IMF's Economist Program
}

\author{
GREG BARRON AND FELIX VÁRDY*
}

This paper shows how the internal job market for participants in the IMF's Economist Program (EPs) could be redesigned to eliminate most of the shortcomings of the current system. The new design is based on Gale and Shapley's (1962) deferred acceptance algorithm and generates an efficient and stable outcome. An Excel-based computer program, EP-Match, implements the algorithm and applies it to the internal job market for EPs. The program can be downloaded from http://www.people.hbs.edu/gbarron/EP-Match_for_Excel.htm. [JEL C78, D73]

he Economist Program is the gate of entry into the IMF for young economists joining the Fund soon after graduate school. Each year, 35 to 45 participants, usually referred to as "EPs," join the program for a two-year period. During this time, EPs work in two different IMF departments. After graduating from the Program, EPs are considered for permanent staff positions.

In the context of the Economist Program, each EP is matched to a department on three occasions. First, upon joining the IMF in June or October; then, upon transfer to a second-year position one year later; and, finally, when the EP is assigned to a permanent position at the end of the program.

Matching of EPs to second-year assignments and permanent positions takes place through a decentralized, internal job market system. Matching of incoming

*Greg Barron is an Assistant Professor at Harvard Business School and Felix Várdy is a second-year participant in the IMF's Economist Program, currently in the Asian Division of the IMF Institute. The authors would like to thank David Coe for encouragement to write this paper, and Abdul Abiad, Chris Clarke, Andrew Feltenstein, Mohsin Khan, Jorge Marquez-Ruarte, Dayalini Mendez, Benoit Mercereau, Miguel Messmacher, Jacques Miniane, Saleh Nsouli, Al Roth, Martin Schindler, Sunil Sharma, Clinton Shiells, Jay Surti, Marijn Verhoeven, Zhiwei Zhang, an anonymous referee, and the editor for valuable comments and suggestions. All errors are our own. 
EPs to first-year positions is done centrally by the IMF's Human Resources Department (HRD).

This paper argues that the procedures currently in place exhibit structural shortcomings. It proposes an alternative centralized matching mechanism, based on Gale and Shapley's (1962) deferred acceptance algorithm (DAA), that resolves most of these shortcomings. Finally, it introduces EP-Match, an Excel-based computer program that implements the DAA and applies it to the internal job market for EPs.

\section{The Current Situation}

\section{Decentralized Matching of Transferring and Graduating EPs}

Transferring and graduating EPs are matched to second-year assignments and permanent positions through a decentralized job market system. However, to promote equality of opportunities, and to prevent unraveling of the market, ${ }^{1}$ departments and EPs are supposed to abide by strict rules as to when they can first contact each other and when they can start making and accepting job offers.

In terms of Roth and Xing's (1994) taxonomy of entry-level labor markets, the internal job market for transferring and graduating EPs is in "Stage 2." Here, "Stage 1" refers to an entirely unregulated job market, while "Stage 3" refers to a fully centralized market. Their detailed description of the symptoms of a typical Stage 2 market is so recognizable that we quote it here at some length.

In stage 2, a $\ldots$ market organization ... attempts to establish a uniform date before which offers should not be made, and often an earlier date before which interviews should not be conducted, and a later date (or time) before which candidates who have received offers should not be required to respond. Sometimes this is hardly successful at all, with many market participants ignoring or circumventing the rules, and those who obey them quickly finding that this puts them at a disadvantage. And even when uniform dates are successfully established and maintained, the market often experiences a great deal of congestion and chaotic behavior, as the deadlines for accepting or rejecting offers grows near. A firm is eager to know if its offers will be accepted in time, so that if it has unfilled positions it may approach its most preferred alternative candidates before they have had to accept any offers they have received. And candidates who have received offers, but not from their first choice firm, are intent upon waiting until the last allowable moment before accepting any offer, in the hope of receiving a better one. Particularly if, as often seems to be the case, some fraction of candidates holds on to multiple offers as the final deadline approaches, this means that just before the deadline expires many transactions still remain to be made. Firms whose first choice candidates reject them may now

\footnotetext{
${ }^{1}$ By "unraveling" we mean the phenomenon, common in entry-level labor markets, of employers making job offers earlier from year to year. This is done in an effort to preempt the competition and recruit the best candidates. Once unraveling has started, it is hard to stop and often spins out of control. In the market for federal appellate law clerks, for example, job offers have recently been made almost two years in advance of employment (see Avery and others, 2001). The same holds for entry-level gastroenterology positions for medical doctors. Interviews for these positions now take place even before students have had the opportunity to explore other subspecialties (see Niederle and Roth, 2003). In markets that experience unraveling, applicants typically receive "exploding" offers that must be accepted or rejected on short notice.
} 
find that their next dozen candidates have already accepted offers, and candidates may receive preferred offers moments after making a verbal commitment to accept an earlier offer. In some markets such verbal commitments are virtually always honored, and in others they are sometimes reneged on. In either event, in the aftermath, many firms and candidates have just missed making connections they would have preferred. The result is that the following year witnesses a resurgence of strategic behaviors designed to avoid being caught short at the end of the market. Often new rules are formulated to prohibit the more brazen of these, and new adaptations are made. While some markets have persisted for many seasons in this fashion, systems of formalized dates are often abandoned, with the market either reverting to stage 1 [an unregulated market], or moving on to stage 3 [a centralized market]." (Roth and Xing, 1994, p. 996.)

From personal experience, one of the authors can attest that the internal job market for transferring and graduating EPs exhibits virtually all the symptoms described by Roth and Xing. Specifically,

- The IMF's Human Resources Department tries to prevent unraveling by enforcing rules on market timing; for example, timing of first contact, interviewing, making, and accepting offers.

- Departments and EPs routinely try to circumvent these rules, while those participants who do obey the rules often end up with rather unfavorable outcomes. A prime example is the rule that EPs and departments should not contact each other before a certain date. This rule is clearly unenforceable.

- When a department makes a job offer to an EP, it often pressures the EP to accept or reject the offer on the spot, or on very short notice.

- Conversely, if EPs can get away with it, they often try to postpone a decision on a particular offer until the very last moment, in the hope of receiving a better offer.

- Sometimes, the hoarding of multiple offers by a few sought-after EPs leads to a virtual standstill in the market. And once these EPs have made up their minds, a flurry of offers and acceptances takes place in a very short time period. (This kind of "congestion" is studied extensively in Roth and Xing, 1997.)

- In response to continuous complaints, HRD has modified the rules governing the market numerous times, apparently without much success.

In addition to the symptoms mentioned by Roth and Xing (1994), it has been noted that

- The current system hurts EPs who are traveling-or prevents departments from sending EPs on business trips during the job market period-because of the limited time frame in which EPs and departments are allowed to interact.

- The current system creates a lot of stress and anxiety among EPs, because they consider the process to be rather unpredictable and not particularly fair.

\section{Centralized Matching of Incoming EPs}

HRD matches incoming EPs to departments on the basis of EPs' and departments' preferences. These preferences are solicited in the form of rank-order lists (ROLs). Participants who do not respond are assumed to be indifferent. 
The process by which HRD translates participants' preferences into a match of EPs and departments is heuristic. This means that HRD tries to find the "best" fit without formulating an explicit objective function or a procedure to resolve conflicting preferences. The drawback of a heuristic approach is that the resulting match is not guaranteed to be Pareto efficient and/or stable. ${ }^{2}$ Indeed, we show in the Appendix that, for a recent cohort of incoming EPs for which we were able to obtain the necessary data, HRD's match was in fact neither.

Motivated by the shortcomings of the matching systems currently in place, in the next section, we discuss Gale and Shapley's deferred acceptance algorithm.

\section{The Deferred Acceptance Algorithm}

\section{Preliminaries}

The internal job market for EPs can be viewed as a "College Admissions Problem" (CAP). The CAP is concerned with two disjoint groups, which we shall refer to as EPs and departments, that have to be matched to each other while taking into account their mutual preferences. The CAP was first formulated by David Gale and Lloyd Shapley in a pathbreaking paper in the American Mathematical Monthly (Gale and Shapley, 1962).

Gale and Shapley (GS) argue that a match of EPs and departments, denoted by $m$, solves the CAP only if it is stable; that is, only if there is no (EP, department) pair that would prefer to be matched to each other over sticking to their matches as prescribed by $m$. Obviously, a matching process that violates this property can easily be upset by an EP and a department getting together in a manner that benefits both. Such an (EP, department) pair is called a "blocking pair." The empirical literature indeed confirms that stability is a very important property in the design of a matching market to fix unraveling and associated Stage 2 market failures (see Table 1, p. 417).

GS prove that, for the CAP, a stable match always exists. ${ }^{3}$ Although there may be multiple stable matches for a given preference profile, when preferences are strict, the set of positions filled and EPs employed are the same in all stable matches (Roth and Sotomayor, 1990). Another attractive feature of stability in the CAP is that it implies Pareto efficiency. ${ }^{4}$ The reverse, however, is not true; that is, typically, there are many Pareto-efficient matches that are unstable. Thus, stability is a strictly stronger requirement than Pareto efficiency.

In their original paper, GS also provide an iterative procedure for finding a stable match with respect to the participants' stated preferences. This procedure is called the deferred acceptance algorithm. Currently, (a variant of) the DAA is used

\footnotetext{
${ }^{2} \mathrm{~A}$ formal definition of stability is given in the next section.

${ }^{3}$ This result is far from trivial, as is illustrated by the closely related "Roommates Problem." In the Roommates Problem, an even number of boys has to be paired up. GS show that, in this case, a stable matching may not exist. Instead, cycles of blocking pairs may cause the set of stable matches to be empty: Consider four boys, A, B, C, and D. Let A rank B first (i.e., B is A's most preferred roommate), while B ranks $\mathrm{C}$ first, and $\mathrm{C}$ ranks $\mathrm{A}$ first. At the same time, A, B, and C all rank D last. Then, regardless of D's preferences, there can be no stable matching; whoever is matched to D will want to move out, and one of the other two will be willing to take him in.

4This follows from the fact that, under strict and "responsive" preferences, bilateral stability implies group stability, and vice versa. See Roth and Sotomayor (1990).
} 
to match applicants and employers in virtually all medical and dental residency programs, pharmacy practices, and psychology internships, both in the United States and Canada (see, e.g., www.nrmp.org). ${ }^{5}$

\section{The Algorithm}

The DAA is best illustrated in a simplified environment in which each department has exactly one vacancy and the total number of vacancies is equal to the total number of EPs. This simple setup is known in the literature as the "marriage problem." Subsequently, we can modify the DAA to handle the general case with an arbitrary number of departments, vacancies per department, and EPs.

It should be noted that, under the DAA, EPs and departments continue to make initial contact and arrange interviews in a decentralized manner. But in the end, instead of making offers directly, participants submit ROLs to the market maker, HRD, which then runs the DAA. The output of the DAA is a stable match; that is, a stable allocation of EPs across departments.

For ease of exposition, we describe the algorithm as if offers and rejections in the DAA are made by the departments and EPs. In reality, it is the market maker that makes these decisions for them, based on the ROLs submitted by the participants.

For the simple case (marriage problem), the DAA works as follows:

Round 0: EPs and departments rank one another in order of attractiveness.

Round 1: Each department makes a job offer to its top-ranked EP. An EP who receives one or more job offers rejects all but the most preferred among the offers received. The relatively most preferred job offer is kept on hold.

Round $i(i \geq 2)$ : A department that was rejected in the previous round makes an offer to its top-ranked EP among those who have not yet rejected it. An EP who receives new offers rejects all but the most preferred among the new offers received and the offer kept on hold from the previous round. Again, the relatively most preferred offer is kept on hold.

Stop: The algorithm terminates when no new job offers are made. At that point each EP has exactly one offer and accepts it.

Note that the roles of EPs and departments in the DAA can be reversed, such that it is the EPs who make the offers to the departments. While both procedures lead to a stable match, the outcomes are not necessarily identical. In fact, Gale and Shapley show that the DAA-generated stable match is preferred over all other stable matches by the side making the offers, provided that preferences are strict.

The extension of the DAA to the general case is now straightforward. With $k$ departments each having a quota of $q_{i}$ positions, $n$ EPs, and EPs making the offers, the algorithm proceeds as follows. First, all EPs apply to the department of their first choice. Department $i$, with $q_{i}$ positions, then places on its waiting list the $q_{i}$ EPs it likes best, or all applying EPs if there are fewer than $q_{i}$. It rejects the rest. Rejected EPs then apply to the department of their second choice. Again, each department selects the top $q_{i}$ among the new applicants and those on the waiting list, puts these

${ }^{5}$ The variant used is the Roth and Peranson (1999) algorithm. Its advantage over the standard DAA is that it can handle couples applying together. 
on its new waiting list and rejects the rest. The procedure terminates when all EPs either are on a waiting list or have been rejected by every department to which they were willing to apply. At this point, each department accepts the offers from everyone on its waiting list and the EP-optimal stable match has been achieved.

Though we have implicitly assumed that all departments rank all EPs (and vice versa), in practice this is not really necessary. Instead, for each of its positions, a department should rank sufficient EPs to be reasonably assured of at least one EP accepting the job. ${ }^{6}$ This implies that the effort spent on interviewing and ranking EPs under the DAA will not differ much from the effort expended under the current system. Also in the current system, a department will want to avoid a situation in which, as a result of too many rejections, it has to start interviewing additional EPs after the market has opened and offers have been made, because, by then, very few EPs will be left to choose from.

Finally, rankings do not have to be strict. Indifference between some or all counterparts is allowed and is resolved by randomization. ${ }^{7}$ Moreover, departments can classify certain EPs as unacceptable, and EPs can do the same for departments. We will get back to these issues later.

\section{Strategic Issues}

\section{Strategy-proofness}

Roth (1982) proves that there is no stable matching mechanism, including the DAA, that is 100 percent strategy-proof. Even in the case of the DAA, it is possible to cook up a constellation of preferences such that at least one participant would gain by distorting them.

The catch is that strategic misrepresentation tends to require much more information about other participants' preferences than is usually available. Roth and Rothblum (1999) show that when participants in the DAA are sufficiently uncertain about the preferences of others, they cannot gain by reversing the order of their true preferences. However, if toward the bottom of the ROL a participant is close to indifferent between being matched or not, and the other side makes the offers, a participant might gain by shortening the ROL, falsely claiming that the least preferred options are "unacceptable." In practice, however, such a strategy is unlikely to be used much, because of its high risk: it might mean that an EP or department ends up with no match at all.

In any case, truncation clearly is not an option for EPs, unless they are prepared to quit and leave the IMF. For departments, truncation is possible only in the market for permanent positions, since, at the end of the day, all transferring EPs have to be matched to a department.

\footnotetext{
${ }^{6}$ For example, in the National Resident Matching Program, each employer interviews and ranks only a tiny fraction of all potential candidates. This does not interfere with the workings of the DAA.

${ }^{7}$ When a large number of participants on both sides of the market are indifferent between many counterparts, a complication may arise. For example, suppose that some EPs and some departments do not care who they are matched to, or have not submitted an ROL. In that case, the DAA proceeds with randomly generated (strict) preferences. What if on the basis of these randomly generated preferences, an indifferent EP and an indifferent department end up matched to each other? Obviously, neither of them can or will
} 
Roth and Rothblum's result of "almost-strategy-proofness in practice" goes a long way in explaining the popularity and robustness of the DAA in real-world applications such as the National Residency Matching Program for physicians. Indeed, Roth (1984a, 1990, 1991) and Roth and Xing (1994) show that, with very few exceptions, centralized matching mechanisms survive if, and only if, they produce stable matches with respect to the participants' (stated) preferences. This is illustrated in Table 1, taken from Roth and Rothblum (1999).

\section{The DAA and the pressure to precommit}

In the current system, departments and EPs routinely flout the rules by committing to one another before the official opening of the market. This raises the question of whether the situation would be any different under the DAA. Specifically, what prevents departments and EPs from reducing the DAA to a mere formality by precommitting to top-rank one another?

In the current system, rejection of an offer in the official market is potentially very costly for a department. The reason is that a rejected department may find that all other attractive EPs have already accepted offers from other departments. To mitigate this risk, departments routinely put extreme pressure on EPs to precommit to accepting a forthcoming job offer. By contrast, rejections are costless in the DAA (at least, from a strategic perspective). The reason is that all acceptances and rejections occur simultaneously. Therefore, a department has no particular interest in making sure that its job offer is accepted by the first EP it is offered to. Indeed, the zero rejection cost in the DAA implies that a department can and should top-rank its favorite EP even if the probability of the EP accepting the offer is very small. In turn, this undermines the credibility of the exploding nature of a pre-market offer, since the department will top-rank its favorite EP, even if the EP refuses to precommit.

These considerations explain why, over time, unraveling and precommitment tend to occur less under the DAA than in decentralized markets. Nevertheless, it is true that precommitment might still occur on a smaller scale, particularly with repeated interaction. In this context, Niederle and Roth (2004b) stress the importance of "market culture," such as norms governing exploding offers.

\section{Departments or positions?}

So far, we have assumed that EPs rank, and are matched to, departments. Usually, however, EPs do not have preferences for departments as such, but for specific positions within departments. For example, even though an EP's first choice might be to work on the China desk in the Asia and Pacific Department (APD), the EP might very well prefer working on South Africa in the African Department over working on Tuvalu in APD. In this case, APD can do two things: either list the

object to that. However, there may be other, non-indifferent EPs or departments that would very much like to be matched to one of these indifferent participants. In that case, we might be able to accommodate one of them without hurting anybody else. In other words, when the randomization is such that indifferent participants end up matched to each other, the matching may not be strictly Pareto optimal. In that case, we should either redo the DAA with newly generated random preferences or manually modify the matching to restore strict Pareto optimality. See the Appendix for a real-world example. 
Table 1. Stable and Unstable Centralized Matching Mechanisms

$\begin{array}{lccc}\text { Market } & \text { Stable } & \text { DAA } & \text { Still in use } \\ \text { Entry level medical markets } & \mathrm{Y} & \mathrm{Y} & \mathrm{Y} \\ \text { US (NRMP) } & \mathrm{Y} & \mathrm{Y} & \mathrm{Y} \\ \text { Edinburgh ('69) } & \mathrm{Y} & \mathrm{Y} & \mathrm{Y} \\ \text { Cardiff } & \mathrm{Y} & \mathrm{Y} & \mathrm{Y} \\ \text { Canada } & \mathrm{Y} & \mathrm{Y} & \mathrm{Y} \\ \text { Cambridge } & \mathrm{N} & \mathrm{N} & \mathrm{Y} \\ \text { London Hospital } & \mathrm{N} & \mathrm{N} & \mathrm{N} \\ \text { Birmingham } & \mathrm{N} & \mathrm{N} & \mathrm{N} \\ \text { Edinburgh ('67) } & \mathrm{N} & \mathrm{N} & \mathrm{N} \\ \text { Newcastle } & \mathrm{N} & \mathrm{Y} & \\ \text { Sheffield } & \mathrm{Y} & & \\ & & & \mathrm{Y} \\ \text { Other markets } & & \mathrm{Y} & \\ \text { Medical specialties } & \mathrm{Y} & \mathrm{Y} & \mathrm{Y} \\ \text { Canadian lawyers } & & \mathrm{Y} & \mathrm{N} \\ \quad \text { Toronto } & \mathrm{Y} & \mathrm{Y} & \mathrm{Y} \\ \quad \text { Vancouver } & \mathrm{Y} & \mathrm{Y} & \mathrm{Y} \\ \quad \text { Calgary and Edmonton } & \mathrm{Y} & \mathrm{Y} & \mathrm{Y} \\ \text { Dental residencies } & \mathrm{Y} & & \mathrm{Y} \\ \text { Pharmacists } & \mathrm{Y} & & \\ \text { Sororities } & \mathrm{Y} \text { (at equilibrium) } & & \end{array}$

China and Tuvalu jobs separately, thereby allowing EPs to express interest specifically in China or specifically in Tuvalu, or lump both jobs together, allowing only for expressions of interest in working for APD, without formal assurance of being assigned to any particular job.

When EPs have preferences for particular positions instead of for departments, there is a trade-off between ex-ante clarity for EPs and ex-post flexibility for departments. If a department chooses to specify its vacancies in great detail, it lowers the risk and/or ambiguity for the applicants. In turn, this may help the department attract better EPs. On the other hand, if a department chooses to be less specific, it retains full flexibility to assign EPs to one job or another, depending on the need of the moment.

Presumably, competition will force all but the most popular departments to list their vacancies separately. From a transparency perspective, separate listings are indeed preferable.

\section{Summary}

The DAA solves many of the problems commonly encountered in matching markets. In the DAA, all final acceptances occur simultaneously. This eliminates premature decisions based on incomplete information. Also, undesirable phenomena such as unraveling, hoarding of multiple offers, and reneging on a prior acceptance will no longer occur. At the same time, it eliminates the need to impose rules on 
the participants that are hard to enforce. In particular, EPs and departments can communicate with one another as early and as often as they wish. The only hard constraint is that participants must hand in their rankings of one another on a preannounced date. The submitted ROLs then determine the matching of EPs to departments (or positions) through application of the DAA. Operationally, a market maker running the algorithm can execute all steps of the DAA in real time, one after the other, provided that EPs and departments have handed in their ROLs. This eliminates the need for repeated communication between EPs and departments, and thereby the need for EPs to be in Washington during any particular time period.

\section{The Computer Program}

\section{Description}

To facilitate implementation of the DAA at the IMF, we have developed an Excelbased computer program, called EP-Match, that executes the algorithm and applies it to the IMF's internal job market for EPs. The user interface of the program consists of four spreadsheets:

1. Instructions (see Figure 1);

2. Departments' Preferences (see Figure 2);

Figure 1. Instructions

Number of EPs:
Dept. Proposing $~$
EP Proposing
2. Fill out the "Departments' Preferences" worksheet according to the instructions on the sheet.
You can customize the departments' and EPs' labels.
3. Indicate the number of open positions for each firm on the "Departments' Preferences" worksheet.
4. Fill out the "EPs' Preferences" worksheets according to the instructions on the sheet.
Here, too. you can customize the departments' and EPs' labels.
5. Click the [Match] button.
The department-optimal stable match will appear in the "Match" spreadsheet. Save or print your
results!
Do not add or remove rows or columns from these four worksheets or change their order.
CLedits:
The der Research fellows - Research Computing Services
Harvard Business School


Figure 2. Departments' Preferences

\section{Departments' Preferences:}

- Each column contains the preference list of one department.

- You can change the names of departments and EPs.

- For each column, enter "1" in the row of the most preferred EP, "2" in the row of the 2nd-most preferred EP, and so on.

- Add a "*" to EP $\times$ 's rank-order to express a department's indifference between EP $\times$ and the EP $y$ ranked one lower than EP $\times$. E.g., "3*" and "4*" in the same column means that the department is indifferent between the EPs (s)he has ranked 3rd, 4th, and 5th.

- Empty cells represent unacceptable EPs.

- Enter the number of positions being offered by each department in the "positions" row.

- Do not add or delete rows or columns from this sheet.

\begin{tabular}{|c|r|r|r|l|}
\hline Clear All & Department 1 & Department 2 & Department 3 & Department 4 \\
\hline \hline Positions & 2 & 2 & 2 & \\
\hline EP1 & $2^{*}$ & 1 & 2 & \\
EP2 & $5^{*}$ & 5 & 4 & \\
EP3 & 1 & 2 & 1 & \\
EP4 & 3 & 3 & 3 & \\
EP5 & 6 & 4 & 5 & \\
EP6 & 4 & 6 & 5 & \\
\hline EP7 & & & & \\
EP8 & & & & \\
EP9 & & & & \\
\hline
\end{tabular}

3. EPs' Preferences (see Figure 3);

4. Match (see Figure 4).

To find the stable match generated by the DAA, the user, that is, the market maker, sequentially works through the four spreadsheets. The first spreadsheet gives instructions on how to use the program and requires the market maker to enter the number of EPs and the number of departments (or positions) participating in the matching process. Here, the market maker must also choose between the EPproposing and department-proposing variant of the DAA. In the second spreadsheet, the market maker specifies the number of vacancies in each department and enters the departments' ROLs in a matrix. The EPs' ROLs go into the third spreadsheet. The fourth and last spreadsheet presents the resulting stable match.

The program is quite versatile. First, it allows for an arbitrary number of EPs, departments, and positions per department. Second, departments are allowed to be 
Figure 3. EPs' Preferences

\section{EPs' Preferences:}

- Each column contains the preference list of one EP.

- You can change the names of departments and EPs.

- For each column, enter "1" for the most preferred department, "2" for the 2nd-most preferred department and so on.

- Add a "**" to department $x$ 's rank-order to express an EP's indifference between department $x$ and the department $y$ ranked one lower than department $x$.

E.g., "1*" means that the EP is in fact indifferent between the department (s)he has ranked 1 st and the department (s)he has ranked 2nd.

- Empty cells represent unacceptable departments.

- Do not add or delete rows or columns from this sheet.

\begin{tabular}{|c|r|r|r|r|r|r|r|r|}
\hline Clear All & EP1 & EP2 & EP3 & EP4 & EP5 & EP6 & \multicolumn{2}{|l|}{ EP7 EP8 } \\
\hline Department 1 & 1 & 1 & 2 & 2 & 1 & 1 & & \\
Department 2 & 2 & 3 & 1 & 3 & 3 & 3 & & \\
Department 3 & 3 & 2 & 3 & 1 & 2 & 2 & & \\
\hline Department 4 & & & & & & & & \\
Department 5 & & & & & & & & \\
Department 6 & & & & & & & & \\
\hline
\end{tabular}

Figure 4. The Match

\section{The Match:}

- Click the [Match] button below.

- The left-hand list is ordered by EP, and the right-hand list is ordered by department.

\begin{tabular}{|c|c|c|c|}
\hline \multirow[b]{2}{*}{ EP } & \multirow[b]{2}{*}{ Department } & \multirow[b]{2}{*}{ Department } & \multirow[b]{2}{*}{ EP } \\
\hline & & & \\
\hline EP1 & $\mathrm{D} 1$ & D1 & EP1 \\
\hline EP2 & D3 & D1 & EP6 \\
\hline EP3 & D2 & D2 & EP3 \\
\hline EP4 & D3 & $\mathrm{D} 2$ & EP5 \\
\hline EP5 & $\mathrm{D} 2$ & D3 & EP2 \\
\hline EP6 & D1 & D3 & EP4 \\
\hline & & & \\
\hline
\end{tabular}


indifferent concerning EPs, and EPs concerning positions. Third, a department can be assured that it will not be matched to an EP it finds unacceptable by not ranking that EP at all. Of course, it should only do this if it prefers leaving the position unfilled to hiring that EP. In principle, EPs can do the same with departments they do not want to join under any circumstances.

Finally, the program can also handle the following, slightly more complex, situation. Suppose department $D x$ has multiple positions open, but its ranking of EPs is not the same for all positions. This might happen if a certain position requires particular skills, such as fluency in a foreign language or financial expertise. In that case, we partition the set of $D x$ 's positions into $r$ disjoint subsets $S_{1}, \ldots, S_{r}$, such that, for all positions in a particular subset, $D x$ 's ranking of EPs is in fact the same. Then department $D x$ is replaced by multiple "virtual departments" $D x_{1}, \ldots$, $D x_{r}$, such that $D x_{i}$ offers positions $S_{i}$ and ranks the EPs accordingly. Finally, the program is run on the modified matching problem.

\section{Examples}

The following two examples illustrate how EP-Match works in practice.

\section{Example 1}

There are three departments, D1, D2, and D3, and six EPs, EP1-EP6. Each department has two vacancies. Let the departments' ROLs be as follows:

\begin{tabular}{l|c|c|c|}
\multicolumn{4}{c}{ Departments' Rankings of EPs } \\
\cline { 2 - 4 } \multicolumn{1}{c|}{} & D1 & D2 & D3 \\
\cline { 2 - 4 } $1^{\text {st }}$ & EP3 & EP1 & EP3 \\
\cline { 2 - 4 } $2^{\text {nd }}$ & EP1 & EP3 & EP1 \\
\cline { 2 - 4 } $3^{\text {rd }}$ & EP4 & EP4 & EP4 \\
\cline { 2 - 4 } $4^{\text {th }}$ & EP6 & EP5 & EP2 \\
\cline { 2 - 4 } $5^{\text {th }}$ & EP2 & EP2 & EP6 \\
\cline { 2 - 4 } $6^{\text {th }}$ & EP5 & EP6 & EP5 \\
\cline { 2 - 4 } & & \multicolumn{3}{|c}{}
\end{tabular}

This means, for example, that D1 likes EP3 best, EP1 second best, and so on.

Similarly, the EPs' ROLs are as follows:

$$
\text { EPs' Rankings of Departments }
$$

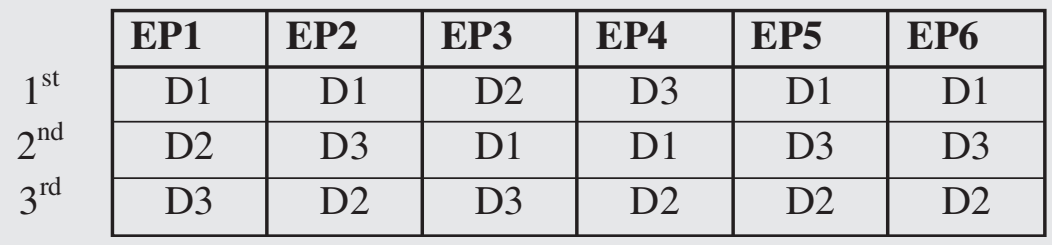

This means, for example, that EP4 prefers the departments in order D3, D1, D2. 
To solve for the associated department-optimal stable match using EP-Match, the market maker first enters the number of departments (3) and EPs (6) in the appropriate boxes on the "Instructions" spreadsheet and then checks that the option "Dept. Proposing" is selected. On the "Departments' Preferences" spreadsheet, the departments' rankings of the EPs are coded by assigning rank-order " 1 " to the mostpreferred EP, "2" to the next-most-preferred EP, and so on. On the same spreadsheet, the number of vacancies ("Positions") in each department (i.e., 2, 2, 2) is entered. The departments' preference matrix then looks like the following:

\begin{tabular}{|c|r|r|r|}
\hline Clear All & D1 & D2 & D3 \\
\hline Positions & 2 & 2 & 2 \\
\hline EP1 & 2 & 1 & 2 \\
EP2 & 5 & 5 & 4 \\
EP3 & 1 & 2 & 1 \\
EP4 & 3 & 3 & 3 \\
EP5 & 6 & 4 & 6 \\
EP6 & 4 & 6 & 5 \\
\hline
\end{tabular}

Similarly, on the "EPs' Preferences" spreadsheet, the market maker codes the EPs' rankings of the departments by assigning rank-order " 1 " to the most-preferred department, "2" to the next-most-preferred, and " 3 " to the least-preferred department. The EPs' preference matrix then looks like this:

\begin{tabular}{|c|r|r|r|r|r|r|}
\hline Clear All & EP1 & EP2 & EP3 & EP4 & EP5 & EP6 \\
\hline \hline D1 & 1 & 1 & 2 & 2 & 1 & 1 \\
D2 & 2 & 3 & 1 & 3 & 3 & 3 \\
D3 & 3 & 2 & 3 & 1 & 2 & 2 \\
\hline
\end{tabular}

Finally, the "Match" button on the "Match" spreadsheet can be pressed, and the department-optimal stable match appears on screen as follows:

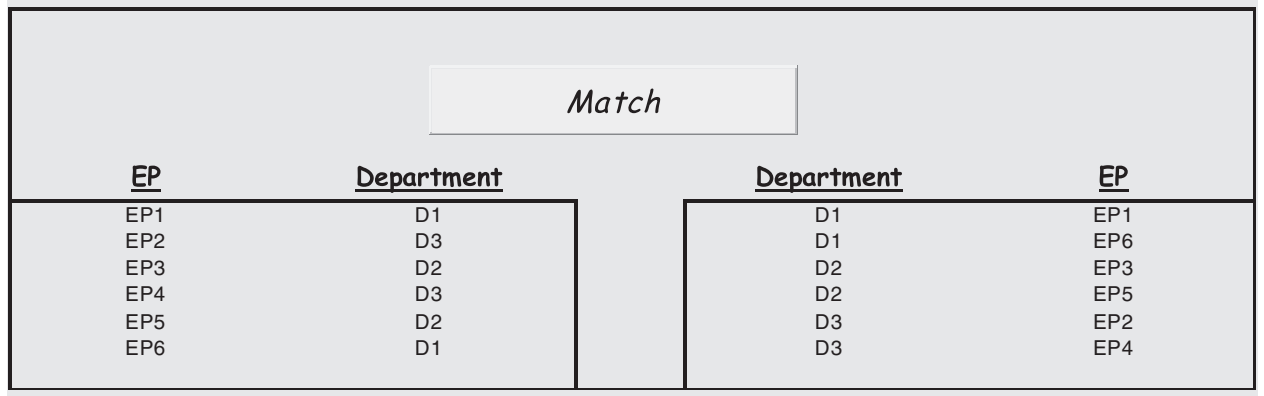


Here, the list on the left is ordered by EP, while the list on the right is ordered by department.

Note that stability implies that no department will be able to lure a more preferred EP from another department. Neither can any EP succeed in "stealing" a more preferred position from another EP.

To calculate the EP-optimal stable match, select the option "EP Proposing" on the Instructions spreadsheet and press the Match button on the Match spreadsheet. It is easily verified that, in this example, the EP-optimal and departmental-optimal stable matches are identical.

\section{Example 2}

In our second example we illustrate how EP-Match can be used to solve more complicated scenarios, when not all EPs are acceptable to all departments, when there are more (or fewer) positions than EPs, and when departments are indifferent about some EPs, and EPs are indifferent about some departments.

Let us take the setup of Example 1 as our point of departure but assume that department D1 is indifferent between EP1 and EP4, and also between EP2 and EP5. Moreover, department D2 now considers EP2, EP5, and EP6 to be unacceptable, while D3 has two additional vacancies (i.e., four instead of two). On the EP side, EP2, EP4, and EP6 are assumed to be indifferent between the department they have ranked second and the department they have ranked third. For the rest, the situation is the same as in Example 1.

In EP-Match, on-screen instructions explain how indifference and unacceptability can be coded into the preference matrices. To express that department $\mathrm{D} i$ is in fact indifferent between EP $x$ and the EP ranked one lower than EP $x$, add a "*" to the rank-order number of EP $x$ on the "Departments' Preferences" spreadsheet. In our example, this means that D1's rank-order numbers of EP1 and EP2, that is, "2" and "5," respectively, get a "*." To express that department D $i$ considers EPy unacceptable, leave cell (EPy, Di) empty. In our example, this means that cells $(\mathrm{EP} 2, \mathrm{D} 2),(\mathrm{EP} 5, \mathrm{D} 2)$, and (EP6, D2) remain empty.

The departments' preference matrix then looks like this:

\begin{tabular}{|c|c|c|c|}
\hline Clear All & D1 & D2 & D3 \\
\hline Positions & 2 & 2 & 4 \\
\hline EP1 & $\overline{2 *}$ & 1 & 2 \\
\hline EP2 & $5^{*}$ & & 4 \\
\hline EP3 & 1 & 2 & 1 \\
\hline EP4 & 3 & 3 & 3 \\
\hline EP5 & 6 & & 6 \\
\hline EP6 & 4 & & 5 \\
\hline
\end{tabular}


Incorporating EP2's, EP4's, and EP6's indifference between their second- and third-ranked departments, we get the following EP preference matrix:

\begin{tabular}{|c|r|r|r|r|r|r|}
\hline Clear All & EP1 & EP2 & EP3 & EP4 & EP5 & EP6 \\
\hline D1 & 1 & 1 & 2 & $2^{*}$ & 1 & 1 \\
D2 & 2 & 3 & 1 & 3 & 3 & 3 \\
D3 & 3 & $2 *$ & 3 & 1 & 2 & $2^{*}$ \\
\hline
\end{tabular}

Now, the department-proposing stable match looks like this:

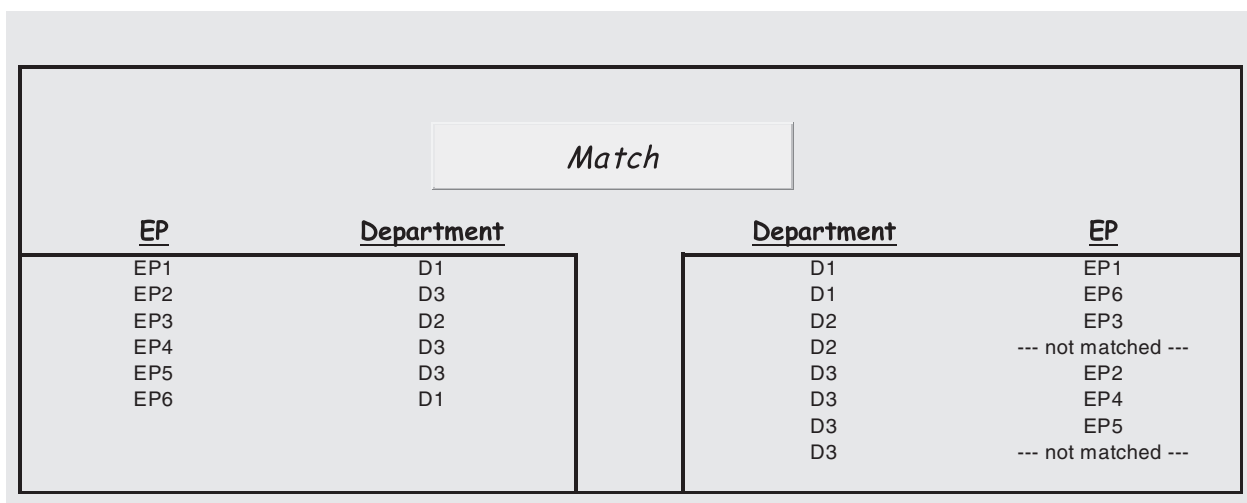

Again, the list on the left is ordered by EP, and the list on the right is ordered by department.

This stable match differs little from the stable match of Example 1. EP5 has moved from D2 to D3, filling one of D3's two additional vacancies, while D2's second and D3's fourth vacancy go unfilled. The latter reflects the fact that, in our example, there are more positions than EPs. The EP-proposing stable match is again identical to the department-proposing stable match.

\section{Conclusion}

In this paper we have argued that adopting the deferred acceptance algorithm to match EPs and departments can enhance the well-being of all parties involved. Unlike the current system, the DAA generates an efficient and stable outcome by optimally using all available information on the preferences of the participants as contained in their rank-order lists. In addition to its attractive theoretical properties, the DAA has been extensively tried and tested in practice, and has produced excellent results. To facilitate implementation of the DAA at the IMF, we have 
developed an Excel-based computer program that executes the algorithm on the basis of EPs' and departments' rankings of each other.

\section{APPENDIX Matching of Incoming EPs}

\section{Introduction}

As described in Section I, the IMF's Human Resources Department (HRD) matches incoming EPs to first-year positions on the basis of EPs' and departments' preferences. The question that arises is whether these matches are stable and/or Pareto efficient. Because HRD tries to find the "best" fit without formulating an explicit objective function or a procedure to resolve conflicting preferences, this question can only be answered by looking at actual data.

In this appendix we show that HRD's match was neither stable nor Pareto efficient in the case of a cohort of incoming EPs for which we were able to retrieve the necessary data. These data consist of the participants' preferences, given by their rank-order lists (ROLs) (Table 2), and the match implemented by HRD (Table 3 ). ${ }^{8}$

\section{Methodology}

Participants (i.e., departments and EPs) who chose not to submit ROLs are assumed to be indifferent as to who they are matched to. Similarly, participants who ranked only a subset of counterparts are assumed to be indifferent about those left unranked, while strictly preferring ranked over unranked counterparts.

To check the stability of HRD's match, it might be tempting to run EP-Match on the participants' preferences and compare the outcomes. However, this would be misguided, because the set of stable matches usually contains many elements, while, in principle, EP-Match finds only two of them; namely, the department-proposing and the EP-proposing stable matches. Thus, discrepancies between HRD's match and those calculated by EP-Match are not necessarily informative.

A second complication arises from the large number of declared and imputed "indifferences" in participants' preferences. Only when preferences are strict does stability imply Pareto efficiency. Otherwise, stable matches may contain "suspect pairs." A suspect pair is a match of an EP and a department, such that both the EP and the department are indifferent between remaining matched to each other and being matched to someone else. If the EP and/or the department making up the suspect pair are very sought after among other departments or EPs, it may be possible to make one or more of these other departments or EPs better off by breaking up the suspect pair, without hurting anybody else. This implies that, with a lot of indifference on both sides of the market, a stable match is only a candidate solution. To be a full-fledged solution, it must be established that none of the suspect pairs give rise to Pareto inefficiencies.

Therefore, a two-step procedure is followed to assess the optimality of HRD's match with respect to the participants' preferences:

- Check the stability of the match by looking for blocking pairs.

- Check whether suspect pairs, if any, give rise to Pareto inefficiencies.

${ }^{8}$ To protect confidentiality we have made certain changes to the data. In particular, "dummy" EPs, departments, and preferences have been added. However, the gist of the example and the validity of the conclusions are fully retained. 


\section{Table 2. Participants' Preferences}

EPs' Preferences over Departments

\begin{tabular}{|c|c|}
\hline EP participant & Preferences ${ }^{1}$ \\
\hline EP1 & $\begin{array}{l}\text { D12, D11*, D13*, D5*, } \\
\text { D7*, D4 }\end{array}$ \\
\hline EP2 & $\begin{array}{l}\mathrm{D} 1, \mathrm{D} 11^{*}, \mathrm{D} 13^{*}, \mathrm{D} 5 *, \mathrm{D} 7 * \\
\mathrm{D} 12^{*}, \mathrm{D} 4\end{array}$ \\
\hline EP3 & No preference indicated \\
\hline EP4 & D1, D6, D13, D12 \\
\hline EP5 & D12, D11 \\
\hline EP6 & No preference indicated \\
\hline EP7 & D1 \\
\hline EP8 & No preference indicated \\
\hline EP9 & No preference indicated \\
\hline EP10 & No preference indicated \\
\hline EP11 & No preference indicated \\
\hline EP12 & D1, D5, D13 \\
\hline EP13 & No preference indicated \\
\hline EP14 & No preference indicated \\
\hline EP15 & D1, D2, D12 \\
\hline EP16 & D1, D3, D10, D13, D5 \\
\hline EP17 & No preference indicated \\
\hline EP18 & D13, D1 \\
\hline EP19 & No preference indicated \\
\hline EP20 & No preference indicated \\
\hline EP21 & D1, D5, D12, D13 \\
\hline EP22 & No preference indicated \\
\hline EP23 & D5 \\
\hline EP24 & D14, D1 \\
\hline EP25 & No preference indicated \\
\hline EP26 & D1, D6, D2 \\
\hline
\end{tabular}

Departments' Preferences over EPs

$\begin{array}{ll}\text { Department } & \text { Preferences }^{1} \\ \text { D1 } & \text { EP7, EP15, EP17 } \\ \text { D2 } & \text { EP12, EP7, EP25 } \\ \text { D3 } & \text { EP19, EP22*, EP11*, EP16 } \\ \text { D4 } & \text { EP22, EP19, EP6, EP11, EP12 } \\ \text { D5 } & \text { EP15, EP21, EP7, EP6, EP19, EP2 } \\ \text { D6 } & \text { EP15, EP1, EP26 } \\ \text { D7 } & \text { EP17, EP14, EP25 } \\ \text { D8 } & \text { No preference indicated } \\ \text { D9 } & \text { No preference indicated } \\ \text { D10 } & \text { EP9*, EP19 } \\ \text { D11 } & \text { EP22, EP6, EP19, EP21, EP23, EP7 } \\ \text { D12 } & \text { No preference indicated } \\ \text { D13 } & \text { No preference indicated } \\ \text { D14 } & \text { E15, EP24 }\end{array}$

${ }^{1}$ Preferences are listed in rank order.

*Indicates indifference between $\mathrm{EP} x$ and the EP ranked one lower than EP $x$.

${ }^{1}$ Preferences are listed in rank order.

*Indicates indifference between department $\mathrm{D} i$ and the department ranked one lower than $\mathrm{D} i$.

Source: IMF's Human Resources Department.

\section{Stability}

Is HRD's match stable with respect to the participants' stated preferences? The answer is no. To see why, note that EP7 and department D1 form a blocking pair: EP7 strictly prefers D1 to his current match D5, and D1 strictly prefers EP7 to its current match EP2.

In this case, a simple exchange is sufficient to transform HRD's match into a stable one. That is, we reassign EP7 to D1 and EP2 to D5, while keeping everything else the same. Both EP7 and D5 go from being matched to a counterpart that was not even on their submitted preference list to being matched to their 1st choice. On the other hand, EP2 goes from his 1st choice to his 2nd choice, while D5 goes from its 3rd choice to its 6th choice. 
THE INTERNAL JOB MARKET OF THE IMF'S ECONOMIST PROGRAM

\section{Table 3. Assignments}

\begin{tabular}{llll}
\multicolumn{2}{c}{ EPs' Departmental Assignments } & \multicolumn{2}{c}{ Departments' EP Assignments } \\
EP & Assigned department & Department & Assigned EP \\
EP1 & D6 & D1 & EP2 \\
EP2 & D1 & D1 & EP15 \\
EP3 & D12 & D1 & EP17 \\
EP4 & D4 & D2 & EP12 \\
EP5 & D11 & D3 & EP16 \\
EP6 & D11 & D3 & EP19 \\
EP7 & D5 & D4 & EP4 \\
EP8 & D11 & D4 & EP11 \\
EP9 & D10 & D4 & EP22 \\
EP10 & D13 & D5 & EP7 \\
EP11 & D4 & D5 & EP21 \\
EP12 & D2 & D6 & EP1 \\
EP13 & D8 & D6 & EP26 \\
EP14 & D7 & D7 & EP14 \\
EP15 & D1 & D7 & EP25 \\
EP16 & D3 & D8 & EP13 \\
EP17 & D1 & D8 & EP20 \\
EP18 & D11 & D10 & EP9 \\
EP19 & D3 & D11 & EP5 \\
EP20 & D8 & D11 & EP6 \\
EP21 & D5 & D11 & EP8 \\
EP22 & D4 & D11 & EP18 \\
EP23 & D11 & D11 & EP23 \\
EP24 & D14 & D12 & EP3 \\
EP25 & D7 & D13 & EP10 \\
EP26 & D6 & D14 & EP24 \\
& & & \\
E5 & & &
\end{tabular}

Source: IMF's Human Resources Department.

\section{Pareto Efficiency}

It is easily established that HRD's match as given in Table 3 contains the following four suspect pairs: (EP3, D12); (EP10, D13); (EP13, D8); and (EP20, D8). Not all of these suspect pairs give rise to Pareto inefficiencies, however. Only the first two suspect pairs do. By breaking up the EP3, D12 pair we can make EP5 strictly better off without hurting anyone else, while breaking up EP10, D13 allows us to make EP18 strictly better off.

Specifically, we perform the following Pareto-improving reassignments:

\section{Box 9}

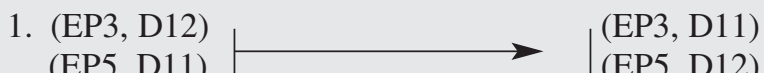

2. (EP10, D13) $($ (EP10, D11)

$(\mathrm{EP} 18, \mathrm{D} 11) \longrightarrow{ }_{(\mathrm{EP} 18, \mathrm{D} 13)}$ 
Greg Barron and Felix Várdy

In this case, EP5 achieves his 1st choice instead of his 2nd choice, while EP18 achieves his 1st choice instead of being assigned to a department not even on his preference list. At the same time, none of the others are worse off than before.

\section{Conclusion}

We have shown that HRD's match of incoming EPs to departments was neither stable nor Pareto efficient. Application of EP-Match followed by a critical assessment of all suspect pairs would have resulted in a better outcome.

\section{BIBLIOGRAPHY}

Avery, C., C. Jolls, R. Posner, and A. E. Roth, 2001, "The Market for Federal Judicial Law Clerks," University of Chicago Law Review, Vol. 68 (Summer), pp. 793-902.

Blum, Y., A. E. Roth, and U. G. Rothblum, 1997, "Vacancy Chains and Equilibration in SeniorLevel Labor Markets," Journal of Economic Theory, Vol. 76, No. 2, pp. 362-411.

Gale, D., 2001, "The Two-Sided Matching Problem: Origin, Development, and Current Issues," International Game Theory Review, Vol. 3, No. 3, pp. 237-52.

— Mathematical Monthly, Vol. 69, pp. 9-15.

Kagel, J. H., and A. E. Roth, 2000, "The Dynamics of Reorganization in Matching Markets: A Laboratory Experiment Motivated by a Natural Experiment," Quarterly Journal of Economics, Vol. 115, pp. 201-35.

Niederle, M., and A. E. Roth, 2003, "Unraveling Reduces Mobility in a Labor Market: Gastroenterology With and Without a Centralized Match," Journal of Political Economy, Vol. 111, No. 6, pp. 1,342-52.

— 2004a, "The Gastroenterology Fellowship Match: How It Failed and Why It Could Succeed Again," Gastroenterology, Vol. 127, No. 2, pp. 658-66.

— 2 2004b, "Market Culture: How Norms Governing Exploding Offers Affect Market Performance," NBER Working Paper No. 10256 (Cambridge, Massachusetts: National Bureau of Economic Research).

Roth, A. E., 1982, "The Economics of Matching: Stability and Incentives," Mathematics of Operations Research, Vol. 7, pp. 617-28.

_ 1984a, "The Evolution of the Labor Market for Medical Interns and Residents: A Case Study in Game Theory," Journal of Political Economy, Vol. 92, No. 6, pp. 991-1016.

— 1984b, "Misrepresentation and Stability in the Marriage Problem," Journal of Economic Theory, Vol. 34, pp. 383-87.

_ 1984c, "Stability and Polarization of Interests in Job Matching," Econometrica, Vol. 52, No. 1, pp. 47-57.

1985, "The College Admissions Problem Is Not Equivalent to the Marriage Problem," Journal of Economic Theory, Vol. 36, pp. 277-88.

, 1986, "On the Allocation of Residents to Rural Hospitals: A General Property of TwoSided Matching Markets," Econometrica, Vol. 54, pp. 425-27.

_ 1989 , "Two Sided Matching with Incomplete Information About Others' Preferences," Games and Economic Behavior, Vol. 1, pp. 191-209.

1990, "New Physicians: A Natural Experiment in Market Organization," Science, Vol. 250, pp. 1524-28. 
1991, "A Natural Experiment in the Organization of Entry Level Labor Markets: Regional Markets for New Physicians and Surgeons in the U.K.," American Economic Review, Vol. 81, No. 3, pp. 415-40.

- 2002, "The Economist as Engineer: Game Theory, Experimental Economics and Computation as Tools of Design Economics," Fisher Schultz lecture, Econometrica, Vol. 70, No. 4, pp. 1341-78.

2003, "The Origins, History, and Design of the Resident Match," Journal of the American Medical Association, Vol. 289 (February), pp. 909-12.

, and E. Peranson, 1999, "The Redesign of the Matching Market for American Physicians: Some Engineering Aspects of Economic Design," American Economic Review, Vol. 89, No. 4, pp. 748-80.

Roth, A. E., and U. G. Rothblum, 1999, “Truncation Strategies in Matching Markets: In Search of Advice for Participants," Econometrica, Vol. 67, No. 1, pp. 21-44.

Roth, A. E., and M. Sotomayor, 1989, "The College Admissions Problem Revisited," Econometrica, Vol. 57, No. 3, pp. 559-70.

_ 1990, "Two-Sided Matching: A Study in Game-Theoretic Modeling and Analysis," Econometric Society Monograph Series (Cambridge, United Kingdom: Cambridge University Press).

— 1992, “Two-Sided Matching," in Handbook of Game Theory, Vol. 1, ed. by R. J. Aumann and S. Hart (Amsterdam: Elsevier), pp. 485-541.

Roth, A. E., and X. Xing, 1994, "Jumping the Gun: Imperfections and Institutions Related to the Timing of Market Transactions," American Economic Review, Vol. 84, No. 4, pp. 992-1044.

_ 1997, "Turnaround Time and Bottlenecks in Market Clearing: Decentralized Matching in the Market for Clinical Psychologists," Journal of Political Economy, Vol. 105, No. 2, pp. 284-329.

Zhou, L., 1991, "Stable Matchings and Equilibrium Outcomes of the Gale-Shapley's Algorithm for the Marriage Problem,” Economics Letters, Vol. 36, No. 1, pp. 25-9. 